Carles Llor ${ }^{1}$

Ana Moragas ${ }^{2}$

Carolina Bayona ${ }^{3}$

Josep M Cots ${ }^{4}$

\title{
Use of clinical vignettes to screen general practitioners who can participate in a clinical trial about stopping antibiotic treatment when this is no longer necessary
}

\author{
${ }^{1}$ Health Centre Barcelona-2B (Via Roma), Spain \\ ${ }^{2}$ Universitat Rovira i Virgili. Health Centre Jaume I, Tarragona, Spain \\ ${ }^{3}$ Health Centre La Marina, Barcelona, Spain \\ ${ }^{4}$ Universitat of Barcelona. Health Centre La Marina, Barcelona, Spain
}

Article history

Received: 1 January 2021; Revision Requested: 1 February 2021; Revision Received: 1 February 2021; Accepted: 12 February 2021; Published: 31 March 2021

\section{Sir,}

Most uncomplicated respiratory tract infections seen in primary care are caused by viruses. Despite being self-limited many patients with these episodes seek care in primary care offices. Inappropriate overuse of antibiotic therapy for these conditions is widespread, with Spain being one of the European countries with the highest consumption, and this overuse being more likely to result in greater spread to the community and individual antibiotic resistance and more adverse effects [1-3]. General practitioners (GP) have generally been told not to discontinue an antibiotic regimen once it has been initiated in order to prevent the patient from acquiring resistant organisms, but this has never been proven. The main objective of the STOP-AB study was to assess if the discontinuation of antibiotic therapy when a GP no longer considers it necessary has any impact on the number of days with severe symptoms. Homogeneity of the possible patients to be included in this trial is crucial and therefore the type of doctors participating in the trial should also be homogeneous, since not all GPs believe that stopping antibiotic therapy is fair and also because not all GPs who wish to participate in a trial such as this consider that antibiotics can be discontinued in the same types of infections. Therefore, we designed a vignette-based questionnaire corresponding to different respiratory tract infections in an attempt to evaluate the rate of agreement among the GPs approached.

A group of GPs from four different autonomous communities in Spain, who had been working for at least 15 years and were confident and comfortable with the strategy of stopping an already commenced antibiotic course filled out a questionnaire with nine vignettes corresponding to patients with different respiratory tract infections. These clinical scenarios were based on a previous study, but they were slightly modified for the present study [4].
The vignettes were differentiated into two different groups: 1. Cases in which antibiotics are clearly indicated: one case of persistent sinusitis (antibiotic indicated), a case of positive-confirmed streptococcal pharyngitis and a case of a radiologically-confirmed pneumonia; and 2. Cases in which antibiotics are not indicated, corresponding to cases of viral pharyngitis, viral rhinosinusitis including the common cold, uncomplicated acute bronchitis and influenza infection. The supplementary material describes the different vignettes used in this study. The study was approved by the Ethical Board of Fundació Jordi Gol i Gurina (reference number: 16/093).

Out of a total of $34 \mathrm{GPs}$ invited to fill out this vignette-based questionnaire, two were excluded as they had been working for less than 15 years and another did not adequately complete the questionnaire. The mean age of the 31 GPs was $50.6 \pm 5.3$ years, with a mean of $24 \pm 5.4$ years of professional work, and $20(64.5 \%)$ were men. The responses are shown in table 1. Only ten GPs (32.3\%) responded appropriately to the nine vignettes and would have included the six patients with suspected viral infection in this randomised clinical trial. One doctor would have included seven patients in the trial, two more would have recruited six patients, albeit incorrectly, whereas the other 18 doctors would have recruited less than six patients.

Antibiotic prescribing varies across countries, across communities and across GPs in the same territory. This study was intended to evaluate the percentage of doctors comfortable and confident with the strategy of discontinuing an already initiated antibiotic course in an attempt to recruit homogenous types of patients with self-limiting respiratory tract infections. The results showed that only one third of GPs gave correct responses and agreed on the types of infections with a likely viral origin, and would, therefore, be candidates to participate in this randomised clinical trial.

Clinical vignettes have a number of advantages for measuring the quality of care provided by doctors. Although it is 


\begin{tabular}{l|cc}
\hline Table 1 & Responses given by the General practitioners (GP) participating in this study \\
& & \\
\hline Clinical scenario & Antibiotics indicated? & Number of GPs who considered discontinuing antibiotic therapy as safe \\
\hline 1. Viral pharyngitis & No & 28 \\
\hline 2. Bacterial rhinosinusitis & Yes & 3 \\
3. Uncomplicated acute bronchitis & No & 14 \\
\hline 4. Streptococcal pharyngitis & Yes & 0 \\
\hline 5. Influenza infection & No & 29 \\
6. Viral rhinosinusitis & No & 28 \\
7. Pneumonia & Yes & 1 \\
8. Viral rhinosinusitis & No & 22 \\
9. Common cold & No & 30 \\
\hline
\end{tabular}

unclear whether vignette responses reflect actual clinical practice or merely doctor knowledge, some studies in clinical care have shown that they can reflect actual behaviour, including acute respiratory tract infections [4-6]. Patients included in a randomised clinical trial must be homogenous and the use of these vignettes has demonstrated the capacity to identify GPS who act similarly in terms of antibiotic prescribing for otherwise uncomplicated respiratory tract infections. We believe these vignettes could be used to quickly identify clinicians who are able to participate in randomised clinical trials on appropriate use of antibiotics in primary care.

\section{FUNDING}

The STOP-AB trial obtained a research grant from the Catalan Society of Family Medicine (Ajut a la recerca CAMFIC 2016).

\section{CONFLICTS OF INTEREST}

$\mathrm{CL}$ reports having received research grants from Abbott Diagnostics. The other authors report no conflicts.

\section{REFERENCES}

1. Goossens $H$, Ferech $M$, Vander Stichele $R$, Elseviers M, ESAC Project Group. Outpatient antibiotic use in Europe and association with resistance: a cross-national database study. Lancet. 2005;365(9459):579-587. DOI: 10.1016/S0140-6736(05)17907-0.

2. Costelloe $C$, Metcalfe $C$, Lovering $A$, Mant D, Hay AD. Effect of antibiotic prescribing in primary care on antimicrobial resistance in individual patients: systematic review and meta-analysis. BMJ. 2010;340:c2096. DOI: 10.1136/bmj.c2096.

3. Shehab N, Lovegrove MC, Geller Al, Rose KO, Weidle NJ, Budnitz DS. US emergency department visits for outpatient adverse drug events, 2013-2014. JAMA. 2016;316(20):2115-2125. D0I: 10.1001/ jama.2016.16201.
4. Gidengil CA, Linder JA, Beach $\mathrm{S}$, Setodji CM, Hunter G, Mehrotra A. Using clinical vignettes to assess quality of care for acute respiratory infections. Inquiry. 2016;53:0046958016636531. DOI: $10.1177 / 0046958016636531$.

5. Evans SC, Roberts MC, Keeley JW, Blossom JB, Amaro CM, Garcia $A M$, et al. Vignette methodologies for studying clinicians' decision-making: Validity, utility, and application in ICD-11 field studies. Int J Clin Health Psychol. 2015;15(2):160-170. DOI: 10.1016/j. ijchp.2014.12.001.

6. Veloski J, Tai S, Evans AS, Nash DB. Clinical vignette-based surveys: a tool for assessing physician practice variation. Am J Med Qual. 2005;20(3):151-157. DOI: 10.1177/1062860605274520. 